\title{
COSTO SOCIAL DEL FREE-RIDE EN CARTAGENA - COLOMBIA: UN ANÁLISIS PARA EL SUBSECTOR DE LOS ESTABLECIMIENTOS DE LAVADO DE VEHÍCULOS
}

\section{SOCIAL COST OF FREE-RIDE IN CARTAGENA - COLOMBIA: AN ANALYSIS FOR THE CAR WASH SUBSECTOR}

\author{
Martha Alicia Yánez Contreras ${ }^{1}$, Flavio Moncayo Flórez ${ }^{2}$, Cindy Paola Otero Betancur ${ }^{3}$ \\ ${ }^{1}$ Economista, M.Sc. Economía, M.Sc. Economía del medio ambiente y recursos naturales, Profesora titular, Directora del \\ Grupo de Investigación Economía y Gestión del Medio Ambiente. Universidad de Cartagena (Colombia), Avenida del consu- \\ lado Calle 30 No48 - 152, e-mail: myanezc@unicartagena.edu.co; ${ }^{2}$ Economista, e-mail: Fr.97@hotmail.com; ${ }^{3}$ Economista, \\ e-mail: Transporte2@actiportsas.com.co
}

Rev. U.D.C.A Act. \& Div. Cient. 19(2): 435-444, Julio-Diciembre, 2016

\section{RESUMEN}

Los establecimientos de lavado de vehículos representan un problema ambiental, debido al uso intensivo del agua, en su funcionamiento. Además, los lavaderos, de la ciudad de Cartagena, tienden a actuar como free-riders, es decir, que en las 15 Unidades Comuneras de Gobierno Urbano, se encuentran lavaderos que evaden parte del pago por el uso de agua, lo que genera un costo, asumido por la sociedad y por las generaciones futuras. El presente artículo, se centra en determinar el costo social producido por el fenómeno del free-ride en los lavaderos de autos en Cartagena, para el periodo 2018, 2028 y 2038. Los resultados obtenidos muestran que el costo social proyectado, para estos lapsos, será de $\$ 1.351$, \$6.051 y $\$ 12.694$ millones a precios corrientes, respectivamente.

Palabras clave: Lavaderos de autos, consumo real, consumo registrado.

\section{SUMMARY}

The vehicle washing facilities represent an environmental problem, due to the intensive use of water as a productive input in their daily operations. Also, the car washes of the city of Cartagena tend to act as free riders, in others words, they evade the full payment for the used water, which generates a cost that is borne by society and future generations. This article focuses primarily on determining the social cost caused by the Free-Ride phenomenon on the car washes from Cartagena for 2018, 2028 and 2038. The results show that the social cost forecasted for those years will be $\$ 1.351$, $\$ 6.051$ and \$12.694 million in current prices respectively.

Key words: Car washes, real consume, registered consume.
Las diferentes decisiones que toman los individuos, a lo largo de su vida, impactan directa e indirectamente en la sociedad en general, inclusive, en las generaciones futuras. En ocasiones, únicamente se tienen en cuenta los costos financieros de una decisión económica, dejando de lado las consecuencias que pueden tener sobre la disponibilidad y la calidad de otros bienes, como la salud, la educación, el medio ambiente, entre otros.

El costo de los daños generados a los bienes es asumido por aquellas personas que hacen uso de los mismos, cuando no es internalizado por la persona que lo produce, es decir, las personas asumen un costo social. La primera persona en acuñar este término fue Pigou (1932), haciendo referencia a la pérdida de bienestar de la sociedad, producto de las deseconomías externas no compensadas, de determinada actividad económica.

Con el paso del tiempo, otros autores han analizado y discutido lo planteado por Pigou, llegando a diversas conclusiones. En primera medida, Coase (1960) menciona que las alternativas propuestas por Pigou, para internalizar el costo social, no son eficientes; únicamente, consideran el detrimento en el bienestar del afectado, sin tener en cuenta el efecto que puede tener, por ejemplo, un impuesto sobre el bienestar del productor del daño. Posteriormente, Lemley (2004) explica que siempre y cuando el productor del daño posea los derechos de propiedad del bien que produce la externalidad, existirá alguna posibilidad de conciliar, antes de imponer una regulación.

Por otro lado, cuando es el propio productor de la externalidad el que internaliza el costo del daño, estimará subjetiva- 
mente un costo menor, ya que es consiente que su actividad económica puede ser gravada (Buchanan, 1999).

Estas proposiciones, se han aplicado en áreas del conocimiento, como la del medio ambiente. Constantemente, las personas y las empresas, al realizar sus actividades económicas, producen efectos desfavorables al medio ambiente, que afectan la calidad de vida de las personas, por ejemplo, la contaminación del aire por las emisiones de carbono.

El costo social producido por esta externalidad ha sido abordado, a través de numerosas alternativas de cálculo, utilizándose, con mayor frecuencia, aquella que supone que los costos marginales de reducción de emisiones representan el costo social del carbono. Las investigaciones de Tol (2008), Anthoff et al. (2009) y Nordhaus (2014) hacen uso de este supuesto metodológico; sin embargo, la primera se basa en una revisión de 211 estimaciones calculadas, previamente, por otros autores.

A diferencia del detrimento de la calidad del aire por las emisiones de carbono existen otros bienes que son afectados por externalidades sin un costo social estimado o monetizado, por ejemplo, el agua.

La cantidad disponible de agua dulce que alberga el planeta tierra, se encuentra limitada, por mantenerse congelada en los polos y glaciares (Serageldin, 1995). Contabilizando el agua congelada, se dice que el mundo tiene alrededor de $8.273 \mathrm{~km}^{2}$ de agua dulce, es decir, agua que puede ser asimilada por el cuerpo humano y así satisfacer sus necesidades básicas (U.S. Geological Survey, 2015).

Esta escasa dotación de agua dulce se ve amenazada, frecuentemente, por diversos factores, como el desperdicio y el mal uso de este recurso, que comprometen su suministro a la población. En respuesta a lo anterior, Petrella (2004), en su manifiesto del agua para el siglo XXI, menciona los principales usos del agua: asegurar la vida y asegurar la existencia de actividades colectivas.

Dentro de las actividades colectivas, se especifican las relacionadas a la producción agrícola, actividades industriales y gestiones de turismo y ocio; sin embargo, existen labores comerciales, como el lavado de autos, que utilizan este recurso de manera intensiva, para satisfacer necesidades, que no son básicas. Este tipo de labores atentan directamente en contra de la disponibilidad y la gestión sostenible del agua, que es un objetivo de obligatorio cumplimiento de cada país (ONU, 2015).

En respuesta a este fenómeno, se han desarrollado estudios de técnicas que se sustentan en la implementación de tecnología avanzada, permitiendo reutilizar el agua consumida
(Brown, 2015). Lastimosamente, en países como Colombia, no se ha dado la implementación de estos sistemas, máxime, por los costos que acarrean.

El Estado, muy seguramente cegado por la premisa cultural que en Colombia este recurso es abundante (Gaitán, 1996), solo regula el uso del agua mediante el cobro de una tasa a toda persona natural o jurídica, que desee satisfacer sus necesidades, mediante el empleo de este bien público (Roy, 2000; Presidencia de la República de Colombia, 2005).

A pesar de lo anterior, Méndez Sayago \& Méndez Sayago (2010) demostraron que esta tasa, aunque incorpora los costos sociales, ambientales y de recuperación del recurso hídrico de donde se extrae el bien, posee diversos problemas y, uno de ellos, es su difícil recaudo. En la ciudad de Cartagena, los lavaderos de autos no son la excepción a la regla. Esta situación genera un costo, que termina siendo asumido por los habitantes de la ciudad, es decir, un costo social.

El agua, por ser un bien público que presenta propiedades de no exclusión y no rivalidad (Samuelson, 1954), induce a que las personas eviten pagar por su uso, ya que otros lo están haciendo por ellos; a este grupo de personas se les denomina free-riders (Hardin, 1971). Otros autores, como Hess \& Ostrom (2006), mencionan que esta situación se produce, no específicamente, cuando una persona evade el pago de un valor monetario por el uso de un determinado bien, sino cuando un individuo, al buscar su interés a expensas de otro, no contribuye con el esfuerzo conjunto realizado por los demás y, al mismo tiempo, recibe beneficios.

La existencia del free-riding en Cartagena es un problema que no debe pasar desapercibido. Mankiw (2002) expresa que el free-ride atenta contra la disponibilidad de cualquier bien público, ya que aquellas empresas que se interesen en ofrecer servicios, como el acueducto o la seguridad, lo harán a niveles sub-óptimos, al no tener suficientes personas que paguen por su uso.

Asimismo, el free-riding es una situación que atenta contra la calidad de los servicios o bienes ofrecidos; un grupo de individuos al no pagar su tiquete de tren, impiden que la empresa obtenga los ingresos presupuestados para y reinvertir en el mejoramiento del servicio, con lo cual, perjudica al resto de beneficiarios (Farieta, 2015).

Otro de las consecuencias que genera el free-riding es que desestimula al resto de beneficiaros del bien a que paguen por el mismo. Todos los consumidores están tentados a evadir el pago del bien público que disfrutan, ya que consideran que su goce no disminuye el stock del mismo, aunque no haya contribuido a su producción (Rawls, 2003). Esto, en el caso de los lavaderos de agua, puede generar que ninguna 
persona esté dispuesta a pagar por el servicio de agua potable o que se dispare el número de lavaderos de agua y que actúen como free-riders.

El presente artículo tiene la finalidad de calcular el costo social que asumirán las generaciones futuras por el free-ride, a partir del análisis de los establecimientos de lavado de vehículos, en la ciudad de Cartagena, desde diferentes escenarios.

\section{MATERIALES Y MÉTODOS}

La presente investigación es de carácter descriptivo, ya que se pretende determinar la magnitud de un fenómeno y su efecto en el tiempo. Este efecto se medirá, a través del uso de fórmulas financieras y así determinar el costo de este fenómeno, en generaciones futuras.

El costo social proyectado, se presenta mediante la agrupación de los lavaderos por Unidades Comuneras de Gobierno Urbano (UCGU). Las UCGU son divisiones administrativas que pertenecen a las localidades del distrito y que, a su vez, agrupan barrios de acuerdo a las características comunes y homogeneidad relativa, de quienes habitan en cada una de ellas. Esta aglomeración, se realiza, principalmente, para tener un mecanismo más robusto de georreferenciación de los lavaderos de la ciudad, de acuerdo los barrios donde están ubicados.

La investigación utilizó información de fuentes secundarias. Se calcula el costo social en la ciudad de Cartagena, a partir de información de encuestas dirigidas a los propietarios o administradores de los lavaderos de vehículos. Asimismo, se empleó información de la empresa Aguas de Cartagena, encargada de la administración y de la regulación del agua; suministró, un listado oficial de los 112 lavaderos de vehículos, registrados en su base de datos y que se encontraban en funcionamiento, al momento de realizar las encuestas. En el directorio que facilitaron, se dio a conocer el consumo de agua, registrado de los últimos meses, de estos establecimientos. De igual manera, se obtuvieron algunos datos e indicadores del Sistema Único de Información de Servicios Públicos (SUI).

Según la ley 240 de 2004, el pago por el consumo de agua, se establece para compensar los costos sociales, ambientales y de recuperación del recurso hídrico. Por tal motivo, el free-ride, al ocasionar que la oferta de bienes públicos se vea afectada por el hecho de no existir suficientes individuos dispuestos a pagar por el consumo, termina generando un costo social.

Para medir el costo social de los free-riders, se procedió a comparar el valor real de consumo de agua de los lavaderos, con el consumo reportado por la entidad administradora de agua de Cartagena. La estimación del costo social se realiza, a través de dos expresiones matemáticas mostradas a continuación:

$$
C s=\left(\sum_{i=1}^{112}(C r-C g)_{i}\right) P e c
$$

Donde, CS es el costo social de los establecimientos de lavado de autos, que actúan como free-riders, expresado en pesos; Pec es la tarifa de agua cobrada a los establecimientos comerciales por metro cúbico que, en 2012, fue de \$3.824 pesos; $\mathrm{Cr}$ es el consumo real de agua de un lavadero de autos; i y $\mathrm{Cg}$ es el consumo registrado por AguaCar del mismo lavadero de autos. Ambas variables de consumo están expresadas en metros cúbicos.

Por otra parte, para el cálculo del costo real del consumo de agua, se efectuó una medición del tiempo necesario para llenar un recipiente de un volumen determinado, empleando las mangueras para lavar los vehículos; el tiempo estimado en cada lavadero junto al volumen del recipiente, componen una magnitud física, denominada caudal. El volumen del recipiente utilizado es de $0,002 \mathrm{~m}^{3}$ y el tiempo estimado en cada lavadero se mide en segundos, por lo tanto, la magnitud resultante, se expresa en $\mathrm{m}^{3} / \mathrm{sg}$.

La medición, se llevó a cabo en abril de 2012. Se visitaron 112 establecimientos de lavado de vehículos, registrados en la empresa AguaCar y se tomó una muestra en cada uno de ellos; en cada establecimiento, se registró el tiempo de lavado de autos particulares, motocicletas, taxis, camionetas y buses. El cálculo del costo real, se resume en la segunda expresión:

$$
C r_{i}=\beta_{i}\left(\sum_{k=1}^{5}(T l \cdot \bar{Q})_{k}\right) D a_{i}
$$

Donde, B es el caudal estimado para un establecimiento de lavado; i expresado en $\mathrm{m}^{3} / \mathrm{sg}$. La estimación de esta variable por cada establecimiento es necesaria para no asumir que la presión de todos los lavaderos es la misma. Algunos establecimientos demoran menos en lavar vehículos y uno de los factores que podría incidir en esta variable es la presión de salida del agua; en este caso, no sería correcto considerar que la diferencia del tiempo de lavado de autos entre un establecimiento y otro se deba completamente a la presión de salida del agua; existen otros factores, tenidos en cuenta en esta investigación ,como el tipo y el tamaño del vehículo y otros no incluidos, como la destreza de la persona encargada de la limpieza o el grado de suciedad presente en los vehículos, antes de ser lavados. Tl es el tiempo de lavado de 
un vehículo; $\mathrm{k}$ y q es el promedio de vehículos $\mathrm{k}$ lavados en un día; Da representa el número de días de atención de un establecimiento i. Por último, $\mathrm{k}$ toma valores de acuerdo al tipo de vehículo que sea lavado: 1 , si es una moto; 2 , si es un auto particular; 3 , si es un taxi; 4 , si es una camioneta y 5 , si es una buseta.

\section{RESULTADOS Y DISCUSIÓN}

La investigación base agrupa los lavaderos de la ciudad de Cartagena por UCGU. La ubicación geográfica de las diferentes Unidades Comuneras de Gobierno, se aprecia en la ilustración 1.

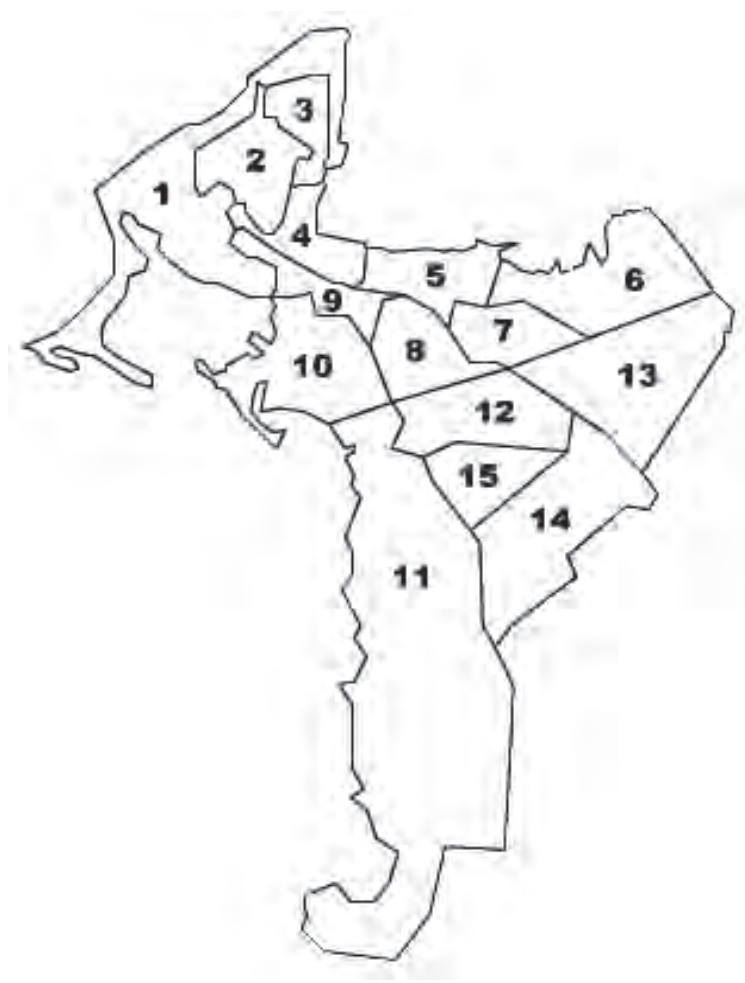

Ilustración 1. Distribución geográfica de las Unidades Comuneras de Gobierno de Cartagena (2009). Fuente: Programa "Cartagena como Vamos".

Además, en la tabla 1, se muestra una caracterización de las UCGU por estrato representativo, número de lavaderos contenidos en ellas y número de autos lavados por semana. Un primer aspecto a destacar es el no registro de lavaderos de vehículos en la comuna 11.

Inicialmente, se podría pensar que, a medida que una comuna albergara mayor número de lavaderos, mayor sería el número de autos lavados semanalmente, en comparación con otra de menor número. La premisa anterior no es estrictamente cierta, ya que en la comuna 13 existen 7 lavaderos y reportaron la limpieza de 103 vehículos, mientras que en la comuna 15, con 4 lavaderos registrados, reportaron 182 carros atendidos.

Una explicación de lo descrito anteriormente es el factor trabajo de cada lavadero y UCGU, representado por las perso- nas destinadas a la limpieza de los autos. Retomando el caso anterior, se podría decir, en primera instancia, que la comuna 15 es más productiva que la comuna 13; la primera, promedia 7,3 personas por lavadero, mientras que la segunda, 5 trabajadores.

A pesar de lo anterior, no se puede atribuir la totalidad de la productividad de un lavadero al factor trabajo. Existen otros aspectos no tenidos en cuenta en esta investigación, como el componente capital.

Por otro lado, en la tabla 2, se muestra el costo social generado por el free-ride de los establecimientos de lavado de autos de la ciudad de Cartagena, en el 2012. 
Tabla 1. Caracterización de los lavaderos de autos de las UCGU de Cartagena (2012).

\begin{tabular}{|c|c|c|c|c|}
\hline UCGU & $\begin{array}{c}\text { Estrato representa- } \\
\text { tivo }\end{array}$ & $\begin{array}{c}\text { Número de lava- } \\
\text { deros }\end{array}$ & Número de trabajadores & $\begin{array}{c}\text { Demanda sema- } \\
\text { nal }\end{array}$ \\
\hline 1 & 5 & 13 & 61 & 457 \\
\hline 2 & 4 & 9 & 58 & 132 \\
\hline 3 & 3 & 4 & 25 & 109 \\
\hline 4 & 2 & 5 & 19 & 115 \\
\hline 5 & 1 & 5 & 28 & 87 \\
\hline 6 & 1 & 8 & 29 & 176 \\
\hline 7 & 3 & 4 & 8 & 281 \\
\hline 8 & 3 & 11 & 52 & 318 \\
\hline 9 & 3 & 12 & 53 & 168 \\
\hline 10 & 2 & 6 & 29 & 527 \\
\hline 12 & 4 & 16 & 75 & 103 \\
\hline 13 & 4 & 7 & 35 & 408 \\
\hline 14 & 1 & 8 & 43 & 182 \\
\hline 15 & 2 & 4 & 29 & 3080 \\
\hline
\end{tabular}

De acuerdo con los resultados, la comuna 9 presenta el mayor costo social generado por el free-ride en Cartagena, con un valor de $\$ 2.581 .697,12$ pesos. Otro aspecto a destacar es la presencia de free-riders en todas las comunas, que regis- traron lavaderos activos. Como se mencionaba anteriormente, la comuna 11 no se muestra en los resultados, ya que no posee lavaderos registrados.

Tabla 2. Costo social mensual del free-ride de los establecimientos de lavado de autos, en Cartagena (2012).

\begin{tabular}{|c|c|c|}
\hline UCGU & CR $-\mathbf{C G}\left(\mathbf{m}^{3}\right)$ & Costo social (pesos) \\
\hline UCG1 & 296,34 & $1.133 .204,16$ \\
\hline UCG2 & 97,79 & $373.948,96$ \\
\hline UCG3 & 89,32 & $341.559,68$ \\
\hline UCG4 & 51,80 & $198.083,20$ \\
\hline UCG5 & 91,02 & $348.060,48$ \\
\hline UCG6 & 331,54 & $1.267 .808,96$ \\
\hline UCG7 & 50,19 & $191.926,56$ \\
\hline UCG8 & 394,83 & $1.509 .829,92$ \\
\hline UCG9 & 675,13 & $2.581 .697,12$ \\
\hline UCG10 & 179,03 & $684.610,72$ \\
\hline UCG12 & 335,99 & $1.284 .825,76$ \\
\hline UCG13 & 61,38 & $234.717,12$ \\
\hline UCG14 & 187,76 & $717.994,24$ \\
\hline UCG15 & 72,20 & $276.092,80$ \\
\hline \multicolumn{2}{|c|}{ Total $2.914,32$} & $11.144 .359,68$ \\
\hline
\end{tabular}


De la misma forma, se detalla que el mayor costo social registrado se destaca en las UCGU que abarcan estratos de clase media, como la 9, 8 y 12. Algunos de los barrios que se encuentran en estas comunas: Chino, Martínez Martelo, El Prado, Amberes, España, Juan XXIII, Paraguay y Junín, en la comuna 9; Zaragocilla, Ángeles, Calamares, Troncal, para la comuna 8 y Caracoles, Blas de Lezo, Campestre y San Pedro, para la 12.

El valor total que se muestra en la tabla 2 es $\$ 11.144 .359,68$ y obedece al costo social producido por lavaderos de autos que actúan como free-riders en Cartagena; sin embargo, este dato solo representa un mes del año, específicamente, abril de 2012. Por consiguiente, se podría decir, que el costo social generado por este fenómeno para un año, se aproximaría a \$133.732.316,16 pesos.

Según los reportes del Sistema Único de Información de Servicios Públicos (SUI), el crecimiento promedio mensual del consumo de los establecimientos comerciales fue de $0,7 \%$, en el 2011. Para este mismo año, el consumo promedio mensual del sector comercial fue de $360.259,33 \mathrm{~m}^{3}$, con una variabilidad del $3 \%$. El bajo porcentaje de variabilidad indica que, en términos generales, el consumo de agua no presenta grandes variaciones de un mes a otro.

El aspecto intergeneracional es una variable importante dentro del análisis del costo social. Cuando una acción produce una externalidad que atenta contra la disponibilidad de un bien, se generarán costos sociales, no únicamente para los individuos que disfrutan, en la actualidad, de dicho bien, sino también a las futuras generaciones, que no podrán disfrutarlo o, por lo menos, no en las mismas condiciones de calidad (Pearce, 2002).

Ejemplo de esto es el costo social ocasionado por los freeriders. Si un gran número de lavaderos de autos decide actuar de manera ilegal y no pagar el valor real de su consumo de agua, ocasionará un déficit, que se verá reflejado en la oferta de bien. Probablemente, en el corto plazo, se siga suministrando el recurso a los individuos; sin embargo, en el largo plazo, no se podrá abastecer a la población creciente de la ciudad, debido a los insuficientes recursos para ejecutar los proyectos de restauración de la cuenca hídrica del canal del dique.

De acuerdo con lo expuesto, es de suma importancia calcular el costo social ocasionado por los lavaderos de autos en el corto y mediano plazo, en la ciudad de Cartagena. Los resultados del primer escenario surgen a partir de los cálculos realizados con la fórmula de valor futuro a pesos corrientes, de una anualidad presentada a continuación:

$$
C s_{t}=C s_{2012} \frac{(1+i)^{t}-1}{i}
$$

Donde, Cst es el costo social generado por los lavaderos que actúan como Free-Rides en un periodo de tiempo; i es la tasa para estimar el costo social en el futuro y $t$, el número de años al que se llevará el valor del costo social actual.

Para el análisis del primer escenario, i toma el valor de 6,25\%, en referencia al incremento promedio anual de las tarifas de consumo de agua del sector comercial, desde el 2004 hasta el 2011. El crecimiento promedio de las tarifas fue calculado a partir de los datos suministrados por el SUI (2016).

Con base a los resultados estimados y presentados en la tabla 3, el costo social mensual incrementará con el paso de los años. Este valor, para el 2018, 2028 y 2038 será, respectivamente, 8, 29, y 66 veces mayor, al reportado en el 2012.

Tal y como es resaltado en el anterior apartado, el impacto del costo social generado por la comuna 9 es amplio. Aproximadamente, el $23 \%$ de todos los ingresos por uso de agua dejados de percibir por la empresa AguaCar, provienen de esta comuna. Es necesario que las autoridades de la ciudad tomen medidas para regular los establecimientos en esta zona y así disminuir el impacto del costo social generado.

Para el segundo análisis, además de aplicar la tasa de crecimiento de la tarifa de consumo de agua, se utiliza la tasa de descuento ambiental gamma, estimada en el trabajo de Correa Restrepo (2008), para simular el desgaste del bien hídrico, a causa de su uso. Esta tasa de descuento tiene la particularidad de no ser constante, ya que hace distinción de acuerdo al número de años que se vaya a evaluar. La tabla 4 muestra las distintas tasas de descuento a utilizar, de acuerdo a determinado plazo.

Para efectuar el segundo cálculo, se utilizó la fórmula número tres, de valor futuro incremental, expresada a continuación:

$$
C s_{t}=C s_{2012} \frac{(1+i)^{t}-(1+g)^{t}}{i-g}
$$

Donde, Cst es el costo social generado por los lavaderos en un periodo; i es una tasa para estimar el valor del costo social en el futuro; t es el número de periodos y g, se tomará como el crecimiento promedio de la tarifa de agua del sector comercial, el cual, fue de 6,1\%, desde el 2004 hasta el 2012. 
Tabla 3. Proyección del costo social mensual del Free-Ride de los establecimientos de lavado de autos, en Cartagena (2018 - 2038).

\begin{tabular}{|c|c|c|c|}
\hline UCGU & $\mathbf{2 0 1 8}$ & $\mathbf{2 0 2 8}$ & $\mathbf{2 0 3 8}$ \\
\hline UCGU1 & 9.584 .743 & 32.687 .029 & 75.045 .897 \\
\hline UCGU2 & 3.162 .894 & 10.786 .477 & 24.764 .589 \\
\hline UCGU3 & 2.888 .942 & 9.852 .215 & 22.619 .624 \\
\hline UCGU4 & 1.675 .405 & 5.713 .667 & 13.117 .964 \\
\hline UCGU5 & 2.943 .927 & 10.039 .729 & 23.050 .136 \\
\hline UCGU6 & 10.723 .243 & 36.569 .676 & 83.960 .035 \\
\hline UCGU7 & 1.623 .332 & 5.536 .080 & 12.710 .243 \\
\hline UCGU8 & 12.770 .278 & 43.550 .718 & 99.987 .756 \\
\hline UCGU9 & 21.836 .228 & 74.468 .496 & 170.971 .643 \\
\hline UCGU10 & 5.790 .499 & 19.747 .448 & 45.338 .013 \\
\hline UCGU12 & 10.867 .172 & 37.060 .522 & 85.086 .964 \\
\hline UCGU13 & 1.985 .258 & 6.770 .364 & 15.544 .027 \\
\hline UCGU14 & 6.072 .860 & 20.710 .389 & 47.548 .821 \\
\hline UCGU15 & 2.335 .217 & 7.963 .837 & 18.284 .112 \\
\hline Total & 94.260 .006 & 321.456 .652 & 738.029 .830 \\
\hline
\end{tabular}

Tabla 4. Esquema de tasas de descuento ambientales para Colombia.

\begin{tabular}{|c|c|}
\hline $\begin{array}{c}\text { Horizonte de evaluación } \\
\text { (años) }\end{array}$ & $\begin{array}{c}\text { Tasa marginal de descuento } \\
\text { (\%) }\end{array}$ \\
\hline $1-5$ & 9,45 \\
\hline $6-25$ & 6,37 \\
\hline $26-75$ & 3,51 \\
\hline $76-100$ & 2,86 \\
\hline Más de 100 & 2,1 \\
\hline
\end{tabular}

Fuente: Extraído de Correa Restrepo (2008).

Las estimaciones, se formalizan para el mismo periodo del primer análisis. Se seleccionaron estos periodos, principalmente los dos últimos, para mostrar la diferencia en el costo social que genera el cambio en la tasa, de acuerdo al plazo.

Conforme a las estimaciones presentadas en la tabla 5, el costo social generado por los free-riders es aún mayor, al tener en cuenta el detrimento ambiental por el consumo de agua, junto al incremento de las tarifas para el sector comercial.

Se observa que para el 2038, la empresa Aguas de Cartagena dejará de percibir $\$ 1.057$ millones, en un solo mes. Por año, esta cantidad ascendería a los \$12.900 millones, aproximadamente. Esta es una cantidad que no podrá ser destinada a la inversión en la conservación de las fuentes hídricas y ampliación de la cobertura de suministro que, sin lugar a dudas, atenta contra el suministro de servicio de agua potable para las generaciones futuras, ya que el agua es un bien insustituible.

Es necesario que las autoridades ambientales de Cartagena y los gobiernos de turno tomen medidas para regular el consumo y las tarifas de agua de estos establecimientos comerciales. En los estratos altos de la ciudad, la tarifa de agua es mayor que aquella que se cobra a establecimientos comerciales.

Una de las conclusiones más generales encontradas dentro del desarrollo de este artículo es el impacto que tiene el free- 
Tabla 5. Proyección del costo social mensual del free-ride de los establecimientos de lavado de autos, en Cartagena, utilizando la tasa de descuento ambiental gama (2018 - 2038).

\begin{tabular}{|c|c|c|c|}
\hline UCGU & $\mathbf{2 0 1 8}$ & $\mathbf{2 0 2 8}$ & $\mathbf{2 0 3 8}$ \\
\hline UCGU1 & 11.451 .215 & 51.280 .057 & 107.565 .590 \\
\hline UCGU2 & 3.778 .816 & 16.922 .038 & 35.495 .846 \\
\hline UCGU3 & 3.451 .517 & 15.456 .350 & 32.421 .403 \\
\hline UCGU4 & 2.001 .663 & 8.963 .713 & 18.802 .381 \\
\hline UCGU5 & 3.517 .208 & 15.750 .525 & 33.038 .469 \\
\hline UCGU6 & 12.811 .419 & 57.371 .230 & 120.342 .498 \\
\hline UCGU7 & 1.939 .449 & 8.685 .112 & 18.217 .982 \\
\hline UCGU8 & 15.257 .081 & 68.323 .227 & 143.315 .523 \\
\hline UCGU9 & 26.088 .477 & 116.827 .648 & 245.058 .909 \\
\hline UCGU10 & 6.918 .104 & 30.980 .187 & 64.984 .368 \\
\hline UCGU12 & 12.983 .377 & 58.141 .278 & 121.957 .761 \\
\hline UCGU13 & 2.371 .855 & 10.621 .481 & 22.279 .732 \\
\hline UCGU14 & 7.255 .450 & 32.490 .867 & 68.153 .186 \\
\hline UCGU15 & 2.789 .963 & 12.493 .825 & 26.207 .179 \\
\hline Total & 112.615 .601 & 504.307 .544 & 1.057 .840 .834 \\
\hline
\end{tabular}

ride sobre el bienestar de la sociedad. El agua es un bien elemental y básico para la vida de todo ser vivo y los lavaderos de autos están generando un costo para la sociedad, poniendo el riesgo la disponibilidad de este bien.

Por otro lado, se puede decir, que el costo social generado por los lavaderos de agua podría ser mucho mayor al calculado. Este trabajo incluye el costo que se genera al actuar como free-riders y no pagar el valor real por el uso de este bien; sin embargo, existe un costo por el hecho de emplear un bien, sumamente escaso, en una actividad que no es esencial, es decir, un costo de oportunidad por utilizar agua potable para lavar carros; no obstante, este valor no entra en el objetivo de este trabajo y, por ende, no fue monetizado.

Cabe resaltar que el costo calculado es un valor que dejará de percibir la empresa AguaCar, al momento de recaudar la prestación de sus servicios; sin embargo, la pérdida principal es asumida directamente por la sociedad, ya que se pone en riesgo la oferta de este bien, para las generaciones futuras

Otro aspecto a destacar es el costo social que asumirán las generaciones futuras por este fenómeno. Para el 2018, el agua será un bien más escaso y más costoso que hoy en día, por ende, este valor, que tendrá que asumir la sociedad, será aproximadamente un $700 \%$ mayor, que el costo de 2012 .
El free-riding, tal y como lo definen Hardin (1971) y Buchanan (1999), es una situación indeseable en cualquier sistema, por lo que los esfuerzos deben ir orientados a determinar algún tipo de mecanismo, que evite la aparición de esta conducta y así se maximicen los beneficios, de aquellos que intervienen en la acción colectiva.

Con base en lo anterior y a los resultados encontrados, las autoridades de la ciudad de Cartagena deben establecer acciones que permitan controlar la evasión en el pago por consumo de agua de los lavaderos de autos. Una medida que podría ser implementada sería establecer un proceso de revisión periódica a los medidores de estos establecimientos, por parte de la empresa AguaCar. Como segunda recomendación, la empresa AguaCar debería implementar sistemas que permitan la comercialización y el consumo de agua no potable, para actividades que no son indispensables para el ser humano; de esta manera, se disminuiría el riesgo del cese de la oferta de este servicio y el desperdicio de agua potable.

Del mismo modo, sería recomendable que estos negocios utilizaran, en su ejercicio diario, otro tipo de agua disponible, como el agua cruda, que se encuentra en su estado natural, sin ningún tipo de tratamiento. Este tipo de líquido es comercializado por AguaCar, a una tarifa de \$3.314, por metro cubico. 
Una recomendación realizada por Kobayashi \& Nakajima (2008), que minimizaría el free-riding generado por la naturaleza de los bienes públicos, es la monetización de los mismos, en este caso, del agua. Los investigadores proponen que el gobierno debe emitir dinero en términos de bienes públicos que, a su vez, se encuentren respaldados con la reserva de los mismos. De acuerdo con ello, el Estado procederá a ajustar la oferta monetaria, de tal manera que ésta y las reservas sean iguales, a un ratio predeterminado de reservas.

Conflictos de intereses: El manuscrito fue preparado y revisado con la participación de todos los autores, quienes declaramos que no existe conflicto de intereses que ponga en riesgo la validez de los resultados presentados.

\section{BIBLIOGRAFÍA}

1. ANTHOFF, D.; HEPBURN, C.; TOL, R. 2009. Equity weighting and the marginal damage costs of climate change. Ecological Economics. 68:836-849 Disponible desde Internet en: http://basesdedatos.unicartagena.edu.co:2053/science/article/pii/ S0921800908002991 (con acceso 01/10/2015).

2. BROWN, C. 2015. International CarWash Association. Disponible desde Internet en: interhttp://www. carwash.org/docs/default-document-library/waterconservation-in-the-professional-car-wash-industry-. pdf?sfvrsn $=0$ (con acceso 01/12/2015).

3. BUCHANAN, J. 1999. Cost and Choise: An inquiry in economic theory. Liberty Found (Indianapolis). 120p.

4. COASE, R. 1960. The problem of social cost. J. Laws and Economics. (Chicago). 3:1-44.

5. CORREA RESTREPO, F. 2008. Tasa de descuento ambiental Gamma: Una aplicación para Colombia. Lecturas de Economía (Medellin). 69:143-162.

6. FARIETA, A. 2015. Aproximaciones éticas al problema del free rider: consecuencialismo, deontología y ética de la virtud. Discuciones Filosóficas (Manizales). 27:147-161.

7. GAITÁN, F. 1996. Cálculo de la tasa por el uso del agua. Planeación y Desarrollo (Bogota). 12:127-158.

8. HARDIN, R. 1971. Collective action as an agreeable nprisoners' dilema. Behavioral Science. 16:472-481. Disponible desde Internet en: http://onlinelibrary.wiley.com/wol1/doi/10.1002/bs.3830160507/abstract (con acceso 27/09/2015).
9. HESS, C.; OSTROM, E. 2006. Understanding Knowledge as a Commons. The MIT Press (Cambridge). 367p.

10. KOBAYASHI, K.; NAKAJIMA, T. 2008. Monetization of public goods provision: a possible solution for the free-rider problem. RIETI. 8:2-12.

11. LEMLEY, M. 2004. Property, intellectual property, and free riding. Tex L. Rev. (Texas). 83:1031.

12. MANKIW, N. 2002. Principios de Economía. McGraw-Hill (Madrid). 811p.

13. MÉNDEZ SAYAGO, J.A.; MÉNDEZ SAYAGO, J.M. 2010. Tasas por utilización del agua ċInstrumento de asignación eficiente del agua o mecanismo de financiación de la gestión ambiental? Estudios gerenciales (Cali). 26:90-115.

14. NORDHAUS, W. 2014. Estimates of the social cost of carbon: Concepts and results from the DICE-2013R model and alternative Aapproaches. J. Assoc. Envirom. Resource Economists (Madison). 1:273-312.

15. ORGANIZACIÓN DE NACIONES UNIDAS -ONU-. 2015. Transofrmar nuestro mundo: la agenda 2030 para el desarrollo sostenible. Disponible desde Internet en: Interhttp://www.un.org/es/comun/docs/?symbol=A/ RES/70/1 (con acceso 21/10/2015).

16. PEARCE, D. 2002. The social cost of carbon and its policy implications. University College of London. Disponible desde Internet en: http://www.ucl. ac.uk/ uctpa15/SOCIAL_COST_OF_CARBON.pdf (con acceso 14/02/2016).

17. PETRELLA, R. 2004. El manifiesto del agua para el siglo XXI. Disponible desde Internet en: https://www. zaragoza.es/contenidos/medioambiente/cajaAzul/ palabras/Petrella_ES.pdf (con acceso 05/01/2016)

18. PIGOU, A. 1932. The Economics of Welfare. London: Macmillan. 872p.

19. PRESIDENCIA DE LA REPÚBLICA DE COLOMBIA. 2005. Decreto 4742 de 2005. Disponible desde Internet en: http://www.alcaldiabogota.gov.co/ sisjur/normas/Norma1.jsp?i=18725 (con acceso 30/12/2015). 
20. RAWLS, J. 2003. Teoría de la justicia. The Belknap Press of Harvard University Press (Cambridge). 549p.

21. ROY, R. 2000. Sustainable product-service systems. Futures [En línea]. 32:289-299. Disponible desde Internet en: http://basesdedatos.unicartagena.edu. co:2053/science/article/pii/S0016328799000981 [consultado el 05/09/2015].

22. SAMUELSON, P. 1954. The pure theory of public expenditure. Review Economics Statistics (Cambridge). 36:387-389.

23. SERAGELDIN, I. 1995. Toward sustainable management of water resources. Banco Mundial (Washigton). 33p.
24. SUI - Sistema Único de Información de Servicios Públicos. 2016. Acueducto. Disponible desde Internet en: www.sui.gov.co/SUIAuth/logon.jsp (con acceso 30/07/2016). /

25. TOL, R. 2008. The social cost of carbon: trends, outliers and catastrophes. Economics: The Open-Access, Open-Assessment E-Journal (Hidenburgufer). 2:125.

26. U.S. GEOLOGICAL SURVEY. 2015. The world's water. Disponible desde Internet en: http://water.usgs.gov/ edu/earthwherewater.html (con acesso 07/08/2015)

Recibido: Mayo 2 de 2016

Aceptado: Octubre 26 de 2016

Cómo citar:

Yánez Contreras, M.A.; Moncayo Flórez, F.; Otero Betancur, C.P. 2016. Costo social del free-ride en Cartagena - Colombia: un análisis para el subsector de los establecimientos de lavado de vehículos. Rev. U.D.C.A Act. \& Div. Cient. 19(2): 435-444. 